\title{
Discrimination of Korean Cattle (Hanwoo) with Imported Beef from USA Based on the SNP Markers
}

\author{
Jung-Mi Shim, Dong-Won Seo, Seongwon Seo ${ }^{1}$, Jong-Joo Kim², \\ Dong-Myung $\mathrm{Min}^{3}$, Ik-Chul Kim ${ }^{3}$, Jin-Tae Jeon ${ }^{4}$, and Jun-Heon Lee* \\ Department of Animal Science and Biotechnology, College of Agriculture and Life Sciences, \\ Chungnam National University, Daejeon 305-764, Korea \\ ${ }^{I}$ Department of Animal Biosystem Sciences, College of Agriculture and Life Sciences, \\ Chungnam National University, Daejeon 305-764, Korea \\ ${ }^{2}$ School of Biotechnology, Yeungnam University, Gyeongsan, Gyeongbuk 712-749, Korea \\ ${ }^{3}$ Experiment \& Research Institute, NAQS, MIFAFF, Seoul 150-804, Korea \\ ${ }^{4}$ Division of Applied Life Science, Gyeongsang National University, Jinju 660-701, Korea
}

\begin{abstract}
Due to the large amount of beef imported from the USA to Korea, Korean consumers have become increasingly interested in the country of origin since it can affect market prices. Previously, Bos indicus and Bos taurus-specific markers were developed for the purpose of cattle breed identification, specifically discrimination of Australian beef. In this study, six SNP markers derived from Illumina 50K bovine SNP chip data were used for the discrimination between Korean cattle (Hanwoo) and imported beef from USA. PCR-RFLP genotyping methods were also developed, which indicates that these markers can be applied relatively easily compared to other markers. Taking into account a discrimination rate of $55 \%$ based on MC1R marker between Hanwoo and imported beef from USA, two additional markers, SNPs 23803 and 34776, were ideal and resulted in probability of identification of 0.942 and probability of misjudgment of 0.03 . Therefore, the markers developed in this study can greatly contribute to the correct discrimination between beef from USA and Hanwoo beef.
\end{abstract}

Key words: MC1R, Korean cattle (Hanwoo), SNP marker, PCR-RFLP, discrimination

\section{Introduction}

The Korean cattle (Hanwoo) are well-known native cattle in Korea and the recorded population is about 2.6 million heads (Ministry for Food, Agriculture, Forestry and Fisheries in Korea, 2009). Along with the recent economic growth, the dietary life has been dramatically changed in Korea and therefore consumers pay more attention to the quality of the food that they have. In Korea, the price of Hanwoo meat is about 2-3 times higher than that of imported beef. Sometimes the beef sellers sell inexpensive imported beef with a label of expensive Hanwoo beef. Because of the mislabeled beef in the marker, Korean consumers like to know the origin

*Corresponding author: Jun-Heon Lee, Department of Animal Science and Biotechnology, College of Agriculture and Life Sciences, Chungnam National University, Daejeon 305-764, Korea. Tel: 82-42-821-5779, Fax: 82-42-825-9754, E-mail: junheon@cnu.ac.kr of the beef that they have and this need makes to develop the DNA markers in order to identify the origin of the beef. Basically, Korean consumers prefer Hanwoo meat rather than imported one, mainly due to the high meat quality. Since the bovine spongiform encephalopathy (BSE) outbreak in the United States in 2003, consumers want to know whether the beef has been imported from USA. For the demand from the consumers, Korean government has started a traceability system in Hanwoo industry in 2009 and now all the cattle have been registered and consumers can trace the beef easily with personal computers. However, this traceability system is not fully settled down and DNA markers are ultimately needed in order to distinguish Hanwoo from imported beef.

Previously, three markers from two mitochondrial genes (ND4, ND5) and a SRY gene, based on the Bos indicus and Bos taurus specific nucleotides, were developed for the cattle breed identification in Japan (Sasazaki et al., 2007). These markers were also successfully used 
for discrimination of Hanwoo beef and imported beef from Australia (Yoon et al., 2008). On the other hand, markers have been developed from the phenotypic appearance such as coat color (Kim et al., 2002). In mammals, the coat color is determined by the action of eumelanin and pheomelanin. Several studies have been reported that the control of Extension (E) and Agouti (A) loci affect coat color variation (Adalsteinsson et al., 1995; Hearing and Tsukamoto, 1991). Recently, a few coatcolor related genes have been investigated including $T Y R$, TYRP1, DCT and MC1R in cattle (Guibert et al., 2004; Gan et al., 2007; Mohanty et al., 2008). Among them, MC1R (Melanocortin 1 receptor) marker has been widely used for distinguishing the cattle breeds having black (for example, Angus and Holstein) and yellow (for example Hanwoo) coat colors.

The purpose of this study is to find efficient breed identification markers, focusing on the discrimination between Hanwoo beef and beef from USA, whose main beef breeds are Angus and Hereford. Since the Angus beef can effectively identified using the MC1R marker, the primary concern of this study was to develop DNA markers for distinguishing Hanwoo from the Hereford breeds.

\section{Materials and Methods}

\section{DNA samples}

The DNA samples from 154 imported meats from USA were used in this study. They were collected by National Agricultural Products Quality Management Service (NAQS) from markets. As the control, 194 Hanwoo (Korean cattle) DNA samples were also used for the allele comparison. They were from three different geographical locations including Seosan (38 $8^{\text {th }}$ candidate bulls, 39 animals), Jinju (Gyeongsang National University, 147 animals) and Daejeon (Chungnam National University, 8 animals) areas. DNA samples were extracted either from muscle or blood using PrimePrep ${ }^{\mathrm{TM}}$ Genomic DNA Isolation Kit (GenetBio, Korea) and DNeasy ${ }^{\circledR}$ Blood \& Tissue Kit (QIAGEN, Germany).

\section{Selection of SNPs}

In order to select the breed specific SNP markers, Illumina 50K bovine SNP chip (Illumina, USA) data were used. Basically, the markers have high differences in allele frequencies were selected between Hanwoo and Hereford breeds. More specifically, the SNP markers having zero allele frequencies in Hanwoo and 0.8 to 0.5 allele frequencies in Hereford were selected (Table 1).
Table 1. Selected SNP markers for breed identification between Hanwoo and Hereford from the Bovine $50 \mathrm{~K}$ SNP chip data

\begin{tabular}{rcccc}
\hline \hline \multirow{2}{*}{ SNP ID } & \multicolumn{2}{c}{ Allele frequency } & $\begin{array}{c}\text { Location of } \\
\text { chromosome }\end{array}$ & $\begin{array}{c}\text { SNP position } \\
\text { in Btau_4.0 }\end{array}$ \\
\hline 26462 & 0 & 0.820 & 2 & 66236338 \\
23803 & 0 & 0.748 & 20 & 69519737 \\
38482 & 0 & 0.683 & 27 & 29362648 \\
34776 & 0 & 0.651 & 20 & 4840282 \\
38141 & 0 & 0.594 & 9 & 90879256 \\
9058 & 0 & 0.553 & 29 & 44660778 \\
\hline
\end{tabular}

\section{PCR amplification and sequencing}

Primers were basically designed using the bovine sequence information from UCSC genome bioinformatics (http://genome.ucsc.edu/). The primer sequences were shown in Table 2. PCR (Polymerase Chain Reaction) was consisted of 1X PCR Gold buffer (Applied Biosystems, USA), $1.9 \mathrm{mM} \mathrm{MgCl}_{2}$ (Applied Biosystems, USA), 0.2 $\mathrm{mM}$ each dNTP (GenetBio, Korea), 10 pmol of each primers, 1 unit of AmpliTaq Gold DNA polymerase (Applied Biosystems, USA) and $20 \mathrm{ng}$ of DNA in a final volume of $20 \mu \mathrm{L}$. The PCR amplification conditions were included five steps with initial denaturation for $10 \mathrm{~min}$ at $94^{\circ} \mathrm{C}$ followed by 35 cycles of $30 \mathrm{~s}$ at $94^{\circ} \mathrm{C}, 30-45 \mathrm{~s}$ at $58^{\circ} \mathrm{C}, 30$ $\mathrm{s}$ at $72^{\circ} \mathrm{C}$ and a final extension at $72^{\circ} \mathrm{C}$ for $10 \mathrm{~min}$. Reaction was performed using either GeneAmp PCR system 2700 (Applied Biosystems, USA) or $\mathrm{C} 1000^{\mathrm{TM}}$ Thermal Cycler (BioRad, USA). After finishing the PCR reaction, the products were visualized in $1.5 \%$ standard agarose gels stained with ethidium bromide (GenetBio, Korea). Each PCR fragments were purified using AccuPrep PCR Purification Kit (Bioneer, Korea). Purified PCR products were sequenced using the same primers for PCR reaction in an automated 3730 XL DNA Sequencer (Applied Biosystems, USA). The DNA sequences were analyzed with Chromas program ver. 2.33 (http://www.technelysium. com.au/chromas) and the SNPs were confirmed based on the electrophoregram results.

\section{PCR-RFLP for identifying alleles}

The PCR-RFLP for identifying MC1R alleles were described previously (Kim et al., 2000) and this method was applied in this study. The PCRs for identifying SNP alleles were performed with the primers for amplifying the genomic DNA segment including the SNP location and alleles were identified using restriction enzymes (Table 2). RFLP reaction was consisted of $1 \times$ buffer, 2 units of each enzymes and $15 \mu \mathrm{L}$ PCR product in a total 
Table 2. PCR primer sequences and restriction enzymes for PCR-RFLP

\begin{tabular}{|c|c|c|c|c|}
\hline SNP name & Primer name & Primer sequence & Product size (bp) & Restriction enzyme \\
\hline 26462 & $26462-05$ & $\begin{array}{l}\mathrm{F}^{\mathrm{a}} \text {-GCCCTCAGAGATGCCTGTTA } \\
\mathrm{R}^{\mathrm{b}} \text {-TCAGGTGGTAATGCGAGTGA }\end{array}$ & 392 & $H p h \mathrm{I}$ \\
\hline 23803 & 23803-01 & $\begin{array}{l}\text { F-ACTAGACGCACAGGCTGCTT } \\
\text { R-GGTGATTCTCCTGACTTTAATGG }\end{array}$ & 309 & BsrGI \\
\hline 38482 & $38482-01$ & $\begin{array}{l}\text { F-CGAAATTTGGTGCATGTTTG } \\
\text { R-CAGCCGCTGACTTAACATGA }\end{array}$ & 665 & Cac $8 \mathrm{I}$ \\
\hline 34776 & $34776-01$ & $\begin{array}{l}\text { F-GCCAAGATGCCAAACTTGAT } \\
\text { R-ATGCACAAACCCTGTTCCTC }\end{array}$ & 810 & $S f c \mathrm{I}$ \\
\hline 38141 & $38141-01$ & $\begin{array}{l}\text { F-GCCTAGATTAGGGCCAGGTC } \\
\text { R-CTGGAAGAGTGCCCGTTAAG }\end{array}$ & 652 & $A c i \mathrm{I}$ \\
\hline 9058 & $9058-01$ & $\begin{array}{l}\text { F-CCAACCTAGCCTTCCTCCTC } \\
\text { R-GTGACCCGACTCTGTGGATT }\end{array}$ & 449 & BsrI \\
\hline
\end{tabular}

${ }^{\mathrm{a}}$ Forward primer, ${ }^{\mathrm{b}}$ Reverse primer.

volume of $20 \mu \mathrm{L}$. The obtained RFLP fragments were separated on $3-5 \%$ standard agarose gels and stained with ethidium bromide.

\section{Calculation of identification probability}

In order to select the best marker combinations for discriminating Hanwoo and Hereford breeds, the identification and misjudgement probabilities were calculated based on the procedures described in Sasazaki et al. (2004). This calculation was considered the marker allele frequencies between two populations and gave the possibilities of identification and misjudgement using these marker combinations.

IB
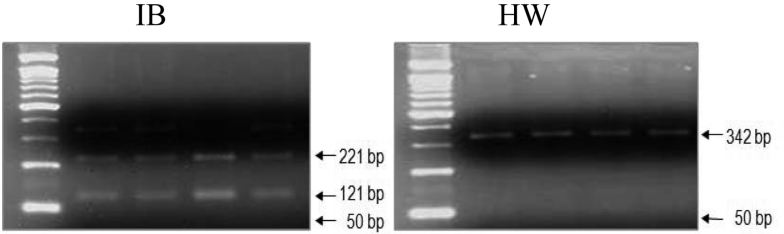

$26462 / \mathrm{Hph}$

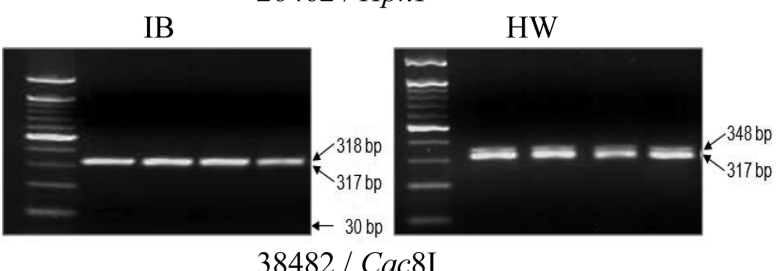

IB
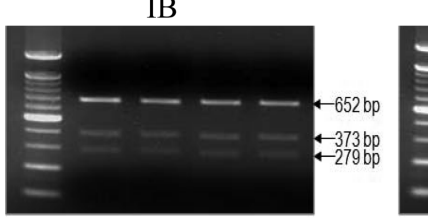

HW

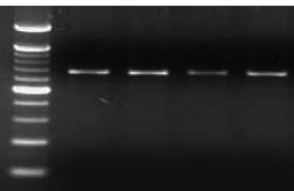

$38141 /$ Aci

\section{Results and Discussion}

Discrimination of imported beef using MC1R marker Initially, 1,037 meat samples from USA were investigated in this study. Since we knew that the one of the major beef cattle breeds in USA is Angus which has the back coat color, the MC1R markers were initially screened for discriminating between Angus (black coat color) and Hanwoo (yellow coat color) breeds. The $350 \mathrm{bp}$ MC1R PCR fragments were digested with two restriction enzymes, namely BsrFI and MspA1I (Klungland et al., 1995). Digestion results with $B s r F I$ can separate the $E^{D}$, $E^{+}$(116 bp and $\left.234 \mathrm{bp}\right)$ types and $e(350 \mathrm{bp})$ type. The undigested $e$ genotype is due to the deletion in guanine at
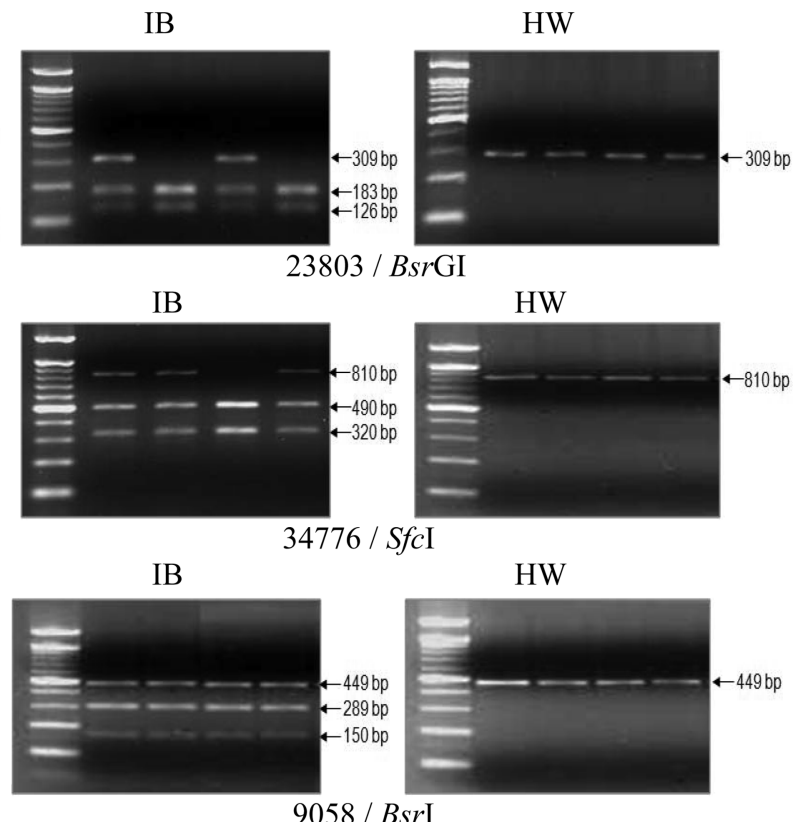

Fig. 1. PCR-RFLP results for the six SNP markers between Hanwoo (HW) and imported beef (IB) samples from USA. In each SNP marker, the restriction enzyme that used for allele identification is also shown. 
Table 3. Genotype and allele frequencies of each SNP markers between Hanwoo and imported beef from USA

\begin{tabular}{|c|c|c|c|c|c|c|c|c|c|c|c|c|}
\hline \multirow{3}{*}{$\begin{array}{c}\text { Marker } \\
\text { name }\end{array}$} & \multirow{3}{*}{$\Sigma$} & \multicolumn{5}{|c|}{ Imported beef from USA } & \multirow{3}{*}{$\Sigma$} & \multicolumn{5}{|c|}{ "Hanwoo } \\
\hline & & \multicolumn{3}{|c|}{ No of Genotype } & \multicolumn{2}{|c|}{ Allele frequency } & & \multicolumn{3}{|c|}{ No of Genotype } & \multicolumn{2}{|c|}{ Allele frequency } \\
\hline & & aa & $\mathrm{ab}$ & $\mathrm{bb}$ & $\mathrm{a}$ & $\mathrm{b}$ & & aa & $\mathrm{ab}$ & $\mathrm{bb}$ & $\mathrm{a}$ & $\mathrm{b}$ \\
\hline 26462 & \multirow{6}{*}{154} & 13 & 85 & 56 & 0.36 & 0.64 & \multirow{6}{*}{194} & 1 & 11 & 182 & 0.03 & 0.97 \\
\hline 23803 & & 34 & 77 & 43 & 0.47 & 0.53 & & 0 & 12 & 182 & 0.03 & 0.97 \\
\hline 38482 & & 0 & 52 & 102 & 0.17 & 0.83 & & 0 & 5 & 189 & 0.01 & 0.99 \\
\hline 34776 & & 19 & 61 & 74 & 0.32 & 0.68 & & 0 & 1 & 193 & 0.003 & 0.997 \\
\hline 38141 & & 0 & 63 & 91 & 0.20 & 0.80 & & 0 & 10 & 184 & 0.03 & 0.97 \\
\hline 9058 & & 6 & 49 & 99 & 0.20 & 0.80 & & 0 & 11 & 183 & 0.03 & 0.97 \\
\hline
\end{tabular}

$310 \mathrm{bp}$ from the beginning of the PCR fragment. On the other hand, RFLP patterns with MspA1I indicated that $E^{D}$ (48 bp, $106 \mathrm{bp}$ and $234 \mathrm{bp}$ ) and $E^{+} / e$ (154 bp and $196 \mathrm{bp}$ ) can be distinguished. The Hanwoo control samples were genotyped for the MC1R markers using the two restriction enzymes and the results show that 184 Hanwoo samples (94.8\%) having ee genotypes and 10 samples (5.2\%) were identified as $E^{+} e$ genotypes. On the other hand, imported meat samples contained $E^{D}$ allele, which is expected from the Angus cattle having black coat color (Kim et al., 2000). The results indicating that 55\% (571/1037) of the USA samples having $E^{D}$ allele for black coat color and this percentage is used for the calculation of identification probability in Table 4 .

\section{Developing the SNP markers}

The selected SNP markers were validated by direct sequencing of PCR fragments containing the SNP locations in Hanwoo and Hereford breeds. For doing this, the SNP information was extracted from the UCSC database including the spanning sequences. For example, the 26462 SNP marker having Thymine in Hereford and Cytosine in Hanwoo from the chip data and the rest five markers, 23803 (A is substituted by G), 38482 (C is substituted by T), 34776 (A is substituted by T), 38141 (C is substituted by $\mathrm{G}$ ) and 9058 ( $\mathrm{T}$ is substituted by $\mathrm{C}$ ), were also confirmed by sequencing.

Table 4. Identification probability and misjudgment probability for the combination of six SNP and MC1R markers. Note that identification probability of the MC1R marker is 0.55

\begin{tabular}{lcc}
\hline \hline \multicolumn{1}{c}{ Marker } & $\begin{array}{c}\text { Probability of } \\
\text { identification }\end{array}$ & $\begin{array}{c}\text { Probability of } \\
\text { misjudgement }\end{array}$ \\
\hline $\mathrm{MC} 1 \mathrm{R}+1^{\mathrm{a}}+2^{\mathrm{b}}+3^{\mathrm{c}}+4^{\mathrm{d}}+5^{\mathrm{e}}+6^{\mathrm{f}}$ & 0.993 & 0.107 \\
$\mathrm{MC} 1 \mathrm{R}+2+3+4$ & 0.960 & 0.041 \\
$\mathrm{MC} 1 \mathrm{R}+2+4$ & 0.942 & 0.030 \\
$\mathrm{MC} 1 \mathrm{R}+3+4$ & 0.875 & 0.014 \\
\hline
\end{tabular}

${ }^{\mathrm{a}} 26462,{ }^{\mathrm{b}} 23803,{ }^{\mathrm{c}} 38482,{ }^{\mathrm{d}} 34776,{ }^{\mathrm{e}} 38141,{ }^{\mathrm{f}} 9058$.
For efficient genotyping of the selected SNP markers, PCR-RFLP methods were developed for each SNP marker. The size ranges of the PCR products are between 309 to 810 bp and the RFLP patterns were easily distinguished using six restriction enzymes, namely $H p h \mathrm{I}, B s r G \mathrm{I}$, Cac8I, SfCI, AciI and BsrI. The 154 imported beef samples were screened by the MC1R markers and these samples do not have $E^{D}$ allele, possibly from Angus breed. In other word, these samples will be more chance to having yellow coat color, which can be from Hereford and Limousine breeds in USA. All six markers developed in this study show clear RFLP patterns and the variations in both Hanwoo and imported beef samples were observed, indicating all of the markers can be efficiently used for discriminating between the samples (Fig. 1).

\section{Analysis of allele and genotype frequencies}

PCR-RFLP was performed for identifying the genotype and allele frequencies in both Hanwoo and imported US beef samples (Table 3). The genotyping results indicated that there are differences in allele and genotype frequencies between the Hanwoo and imported beef samples. For example, SNP marker 23803 shows $a$ allele frequency is 0.47 in imported beef samples from USA. On the other hand, this frequency in Hanwoo is 0.03 . Therefore, the observed allele difference for allele $a$ between two populations is 0.44 . With the same method, $a$ allele frequencies in both imported US beef samples and Hanwoo were observed for calculating the allele differences in other markers. These allele frequencies were compared with the $50 \mathrm{~K}$ Illumina chip data. Because we initially selected the markers having large allele frequency differences between the Hanwoo and Hereford, the observed allele frequency differences are much less than we expected. This may indicated that the Hanwoo samples for the chip data could not represent the samples used in this study. The samples for the chip experiments were from the Hanwoo samples in National Institute of Animal Sciences in 
Korea and they are mainly elite Hanwoo individuals. Another reason for these allele differences is that the major breed for the imported beef samples from USA is not Hereford. This may possible because several beef cattle breeds are available in USA, even though the major breeds are Angus and Hereford.

The allele frequencies also indicated that there are differences between the chip data and PCR-RFLP results in Hanwoo samples. We initially selected the SNP markers having the Hanwoo allele frequencies were zero. However, the results indicate that all six makers had $a$ allele frequencies between 0.003 to 0.03 . This indicates that the heterogeneity of the Hanwoo breeds. The similar results were observed in Yoon et al. (2008) that Hanwoo animals have small portion of unexpected Bos indicus alleles.

\section{Identification probability using SNP markers}

The allele differences between imported beef from USA and Hanwoo were used for calculating the probabilities of identification and misjudgment. The Hardy-Weinberg equilibrium test was also performed. SNP markers 26462,38482 , and 38141 were statistically significant $(p<0.05)$. Whereas, the SNP markers 23803, 34776, and 9058 were not significant indicating the selection pressure was not applied in these markers. Including the discrimination percentage $(55 \%)$ of MC1R marker, the highest probability of identification of 0.976 using the six markers. However, this is not an ideal combination due to $10 \%$ probability of misjudgment which were resulted from the accumulation of errors.

Considering the cost of PCR-RFLP experiments, relatively small number of markers is more beneficial. The most efficient marker combinations were shown in Table 4. The results indicated that two SNP markers, 23803 and 34776, in addition to MC1R, is the most ideal combination since the probability of identification was 0.942 and probability of misjudgment was 0.03 . Alternatively, another marker combination including 38482 and 34776 SNPs, is also a feasible combination since this marker combination gave the lowest probability of misjudgment of 0.014 . However, its probability of identification is the lowest among four combinations in Table 4.

\section{Conclusions}

Six SNP markers have been selected based on Illumina chip data for discriminating Hanwoo and the beef samples from USA. Among these, the SNP markers 23803 and 34776 with the powerful MC1R marker information gave the best combination showing 0.942 of the probability of identification and 0.03 of the probability of misjudgment. The markers developed in this study can greatly contribute the identification of beef from USA for giving correct information for the beef consumers in Korea.

\section{Acknowledgements}

This study was financially supported by research fund of Chungnam National University in 2009.

\section{References}

1. Adalsteinsson, S., Bjarnadottir, S., Vage, D. I., and Jonmundsson, J. V. (1995) Brown coat color in Icelandic cattle produced by the loci extension and Agouti. J. Hered. 86, 395-398.

2. Gan, H. Y., Li, J. B., Wang, H. M., Gao, Y. D., Liu, W. H., Li, J. P., and Zhong, J. F. (2007) Allele frequencies of TYR and MC1R in Chinese native cattle. J. Anim. Sci. 78, 484-488.

3. Guibert, S., Girardot, M., Leveziel, H., Julien, R., and Qulmouden, A. (2004) Pheomelanin coat colour dilution in French cattle breeds is not correlated with the TYR, TYRP1 and DCT transcription levels. Pigment Cell Res. 17, 337-345.

4. Hearing, V. J. and Tsukamoto, K. (1991) Enzymatic control of pigmentation in mammals. FASEB J. 5, 2902-2909.

5. Kim, T. H., Yoon, D. H., Park, E. W., Lee, H. Y., Oh, S. J., Cheong, I. C., Thak, T. Y., Kim, K. N., and Han, J. Y. 2000. A study on genotype frequencies of the bovine melanocortin recepter 1 (MC1R) in cattle breeds. J. Anim. Sci. Technol. 42, 735-744.

6. Klungland, H., Vage, D. I., Gomez-Raya, L., Adalsteinsson, S., and Lien, S. (1995) The role of melanocyte-stimulating hormone (MSH) receptor in bovine coat color determination. Mamm. Genome 6, 636-639.

7. Mohanty, T. R., Seo, K. S., Park, K. M., Choi, T. J., Choe, H. S., Baik, D. H., and Hwang, I. H. (2008) Molecular variation in pigmentation genes contributing to coat colour in native Korean Hanwoo cattle. Anim. Genet. 39, 550-553.

8. Sasazaki, S., Itoh, K., Arimitsu, S., Imada, T., Takasuga, A., Nagaishi, H., Takano, S., Mannen, H., and Tsuji, S. (2004) Development of breed identification markers derived from AFLP in beef cattle. Meat Sci. 67, 275-280.

9. Sasazaki, S., Mutohh, H., Tsurifune, K., and Mannen, H. (2007) Development of DNA markers for discrimination between domestic and imported beef. Meat Sci. 77, 161-166.

10. Yoon, D., Kwon, Y. S., Lee, K. Y., Jung, W. Y., Sasazaki, S., Mannen, H., Jeon, J. T., and Lee, J. H. (2008) Discrimination of Korean cattle (Hanwoo) using DNA markers derived from SNPs in bovine mitochondrial and SRY genes. Asian-Australas. J. Anim. Sci. 21, 25-28.

(Received 2010.6.4/Revised 2010.11.1/Accepted 2010.11.3) 